\title{
Design Concept Evaluation Based on Rough Number and Information Entropy Theory
}

\author{
Jie Hu, Guoniu Zhu, Jin Qi, Yinghong Peng \\ School of Mechanical Engineering \\ Shanghai Jiao Tong University \\ Shanghai, China \\ hujie@sjtu.edu.cn
}

\author{
Xiaohong Peng \\ School of Engineering \& Applied Science \\ Aston University \\ Birmingham, UK
}

\begin{abstract}
Concept evaluation at the early phase of product development plays a crucial role in new product development. It determines the direction of the subsequent design activities. However, the evaluation information at this stage mainly comes from experts' judgments, which is subjective and imprecise. How to manage the subjectivity to reduce the evaluation bias is a big challenge in design concept evaluation. This paper proposes a comprehensive evaluation method which combines information entropy theory and rough number. Rough number is first presented to aggregate individual judgments and priorities and to manipulate the vagueness under a group decision-making environment. A rough number based information entropy method is proposed to determine the relative weights of evaluation criteria. The composite performance values based on rough number are then calculated to rank the candidate design concepts. The results from a practical case study on the concept evaluation of an industrial robot design show that the integrated evaluation model can effectively strengthen the objectivity across the decision-making processes.
\end{abstract}

Keywords-design concept evaluation; information entropy; rough number; composite performance value; subjectivity

\section{INTRODUCTION}

With fast changing in customer needs and rapid progress in technology, companies are required to develop new products with shorter time, higher quality and lower cost to maintain competitiveness in a highly competitive global market [1]. However, any new product development is full of uncertainty and risks. To ensure the success of this development and solve the stochastic problems involved, various factors and constraints should be taken into consideration as early as possible for making accurate decision. An improper evaluation of the design concept may not only cause additional modification, but also increase the development cost and cycle or even endanger the entire development process. Therefore, evaluating the design concept at the early stage and using appropriate methods is one of the most vital activities in new product development.

In general, the design concept evaluation is a process of group decision-making, where many decision makers are invited to carry out the performance assessment. Meanwhile, the evaluation information is mainly subjective and uncertain since it largely depends on experts' individual judgments. How to objectively aggregate individual judgments and preferences in the evaluation of the candidate concepts under such a subjective environment becomes a critical issue.

Information entropy theory provides a good example in dealing with uncertainty. Generally, entropy is established on the basis of probability theory and widely used as a measure of uncertainty in the information system. A remarkable superiority of the entropy method is that it can calculate the objective weights without considering the preferences of decision makers. Therefore, to deal with the subjectivity in design concept evaluation, this paper presents a systematic approach to enhancing the objectivity of the evaluation process by utilizing the information entropy theory. Furthermore, rough number is also introduced to aggregate individual judgments and preferences and to manage the vagueness in the evaluation process. Specifically, a rough number based entropy weight method is developed to calculate criteria weights, which are used to generate the corresponding composite performance values to evaluate the candidate design concepts.

The rest of this paper is structured as follows. Related work is reviewed in Section II. Section III presents the integrated rough entropy based evaluation method. Section IV put forwards a practical case study concerning industrial robot design concept evaluation. Finally, the conclusion is drawn in Section V.

\section{RELATED WORK}

\section{A. Information entropy theory in decision-making}

Due to its powerful ability in objective criteria weighting, the entropy weight method has been widely used in various decision-making areas. Yang and Qiu [2] incorporated the expected utility decision theory using the entropy method and developed a decision-making model based on expected utility and entropy. Li et. al [3] presented a safety evaluation model of coal mines by combining the entropy weight method with the TOPSIS approach. The entropy weight method is applied to calculate the relative importance of each index and TOPSIS is used to rank the candidate coal mines. El-Santawy [4] combined the information entropy weight method with VIKOR to solve the multi-criteria decision making (MCDM) problem of the personnel training selection. The information entropy weight method is used in criteria weighting and VIKOR is adopted to conduct the final alternatives ranking. Safari et.al [5] introduced an integrated 
method for supplier selection which employs both Shannon's entropy and PROMETHEE techniques. The entropy is used to determine the relative weight and PROMETHEE is applied to rank the candidate alternatives. Liu and Zhang [6] used entropy weight in combination with an improved ELECTRE-III method for the supplier selection, where entropy weight is used in criteria weighing and ELECTREIII is utilized to arrange the alternatives. $\mathrm{Li}$ et al. [7] also established an entropy weighted osculating value method for groundwater quality assessment.

However, in the most cases, the information provided in design concept evaluation is highly vague and imprecise. A simple crisp approach is unable to capture the true perception of the decision maker. To manage the vagueness in decisionmaking, fuzzy logic is introduced and various fuzzy sets based models are developed.

Kulak [8] developed a decision support system which contains a rule-based system, a database and several MCDM models. The fuzzy information axiom is introduced in its final alternatives selection. To handle the incomplete information in design concept evaluation, Akay and Kulak [9] developed a grey-fuzzy information axiom to evaluate design concepts. Büyüközkan [10] put forward a decision model for supplier performance evaluation by combining an analytical hierarchy process (AHP) with the axiomatic design. Akay et al. [11] presented an interval-type-2 fuzzy information axiom to handle the uncertainty and subjectivity in the concept selection. Weng and Jenq [12] built a hierarchical decisionmaking model by Applying fuzzy information axiom is applied in the equipment evaluation. Cebi and Kahraman [13] developed a group decision support system which contains a knowledge system module, an inference engine module and a user interface module. Based on the fuzzy information axiom and fuzzy AHP, Kulak and Kahraman [14] presented a fuzzy MCDM method and applied it in transportation company selection. Ji et al. [15] integrated the entropy weight method, fuzzy set theory and the MCDM method. By avoiding subjective effects on the weights, the proposed model can assess the MCDM problems in a more objective manner. Liu et. al [16] extended the information entropy method with fuzzy theory to develop an enhanced fuzzy comprehensive evaluation method for water quality assessment. In addition, an information entropy technique has been applied to calculate the coefficient of weight and to exploit the useful information of data to a maximum extent. Chen and Li [17] proposed an objective weighting method which employs intuitionistic fuzzy entropy measures to tackle MCDM problems under the intuitionistic fuzzy sets environment. Zhao et. al [18] applied a new model in the reliability evaluation of power communication networks, where FAHP and entropy method were combined to calculate the weights of the criteria to enhance the credibility and objectivity of the results obtained. Ye [19] suggested a fuzzy MCDM method based on weighted correlation coefficients using the intuitionistic fuzzy entropy weights. This method is particularly useful in the situation where the weights information is completely unknown. He further optimized the entropy weights-based correlation coefficients in terms of the interval-valued intuitionistic fuzzy sets [20].
In summary, the purpose of the fuzzy valued evaluation method is to transform crisp numbers into fuzzy ones to manipulate the vagueness in decision-making. But the determination of the membership function in fuzzy sets is mainly depending on subjective judgments. Most of the fuzzy evaluation methods need to introduce auxiliary information which is subjective as well. To tackle the dilemma, rough number is introduced to deal with the vagueness and subjectivity [21] concerned, as it only depends on the original data and no auxiliary numbers are required.

\section{B. Rough number}

Generally, a rough number is constituted by the rough boundary interval, lower limit and upper limit [22]. Suppose $\mathrm{U}$ is the universe, $\mathrm{R}$ is a set of $\mathrm{n}$ classes $\left(C_{1}, C_{2}, \cdots, C_{n}\right)$ that cover all the objects in $\mathrm{U}, R=\left\{C_{1}, C_{2}, \cdots, C_{n}\right\}, \mathrm{Y}$ is an arbitrary object of $\mathrm{U}$. If these classes are ordered as $C_{1}<C_{2}<\cdots<C_{n}$, then $\forall Y \in U, C_{i} \in R, 1 \leq i \leq n$, the lower approximation $\left(\underline{\operatorname{Apr}}\left(C_{i}\right)\right)$, upper approximation $\left(\overline{\operatorname{Apr}}\left(C_{i}\right)\right)$ and boundary region $\left(B n d\left(C_{i}\right)\right)$ of class $C_{i}$ are defined as:

$$
\begin{aligned}
\underline{\operatorname{Apr}}\left(C_{i}\right) & =\bigcup\left\{Y \in U / R(Y) \leq C_{i}\right\} \\
\overline{\operatorname{Apr}}\left(C_{i}\right) & =\bigcup\left\{Y \in U / R(Y) \geq C_{i}\right\} \\
\operatorname{Bnd}\left(C_{i}\right) & =\bigcup\left\{Y \in U / R(Y) \neq C_{i}\right\} \\
& =\left\{Y \in U / R(Y)>C_{i}\right\} \bigcup\left\{Y \in U / R(Y)<C_{i}\right\}
\end{aligned}
$$

Furthermore, the lower limit $\left(\underline{\operatorname{Lim}}\left(C_{i}\right)\right)$, upper limit $\left(\overline{\operatorname{Lim}}\left(C_{i}\right)\right)$ and rough number $\left(R N\left(C_{i}\right)\right)$ of $C_{i}$ can be defined as:

$$
\begin{aligned}
& \underline{\operatorname{Lim}}\left(C_{i}\right)=\frac{1}{M_{L}} \sum R(Y) \mid Y \in \underline{\operatorname{Apr}}\left(C_{i}\right) \\
& \overline{\operatorname{Lim}}\left(C_{i}\right)=\frac{1}{M_{U}} \sum R(Y) \mid Y \in \overline{\operatorname{Apr}}\left(C_{i}\right) \\
& R N\left(C_{i}\right)=\left\lceil\underline{\operatorname{Lim}}\left(C_{i}\right), \overline{\operatorname{Lim}}\left(C_{i}\right)\right\rfloor
\end{aligned}
$$

where $M_{L}, M_{U}$ are the numbers of objects contained in $\operatorname{Apr}\left(C_{i}\right)$ and $\overline{\operatorname{Apr}}\left(C_{i}\right)$, respectively.

Their difference is defined as the rough boundary interval $\left(\operatorname{IRBnd}\left(C_{i}\right)\right)$ :

$$
\operatorname{IRBnd}\left(C_{i}\right)=\overline{\operatorname{Lim}}\left(C_{i}\right)-\underline{\operatorname{Lim}}\left(C_{i}\right)
$$

The rough boundary interval denotes the vagueness of $C_{i}$, where a larger one means more vague while a smaller one denotes a better precise. The subjective information can then be denoted by rough number. 
Suppose

$$
R N(a)=\lceil\underline{\operatorname{Lim}}(a), \overline{\operatorname{Lim}}(a)\rfloor
$$

and $R N(b)=\lceil\underline{\operatorname{Lim}}(b), \overline{\operatorname{Lim}}(b)\rfloor$ are two rough numbers, $\lambda$ is a nonzero constant, the arithmetic rules can be defined as [23]:

$$
\begin{aligned}
& R N(a)+R N(b)=\lceil\underline{\operatorname{Lim}}(a), \overline{\operatorname{Lim}}(a)\rfloor+\lceil\underline{\operatorname{Lim}}(b), \overline{\operatorname{Lim}}(b)\rfloor \\
&=\lceil\underline{\operatorname{Lim}}(a)+\underline{\operatorname{Lim}}(b), \overline{\operatorname{Lim}}(a)+\overline{\operatorname{Lim}}(b)\rfloor \\
& R N(a) \times \lambda=\lceil\underline{\operatorname{Lim}}(a), \overline{\operatorname{Lim}}(a)\rfloor \times \lambda \\
&=\lceil\lambda \times \underline{\operatorname{Lim}}(a), \lambda \times \overline{\operatorname{Lim}}(a)\rfloor \\
& \begin{aligned}
R N(a) \times R N(b) & =\lceil\underline{\operatorname{Lim}}(a), \overline{\operatorname{Lim}}(a)\rfloor \times\lceil\underline{\operatorname{Lim}}(b), \overline{\operatorname{Lim}}(b)\rfloor \\
& =\lceil\underline{\operatorname{Lim}}(a) \times \underline{\operatorname{Lim}}(b), \overline{\operatorname{Lim}}(a) \times \overline{\operatorname{Lim}}(b)\rfloor
\end{aligned}
\end{aligned}
$$

It will be shown later that using the rough number approach can strengthen the objectivity and capture the decision makers' real perception in various group decisionmaking areas.

\section{PROPOSED METHOD}

\section{A. Framework of the proposed method}

To manipulate the subjectivity and vagueness in design concept evaluation, this paper presents an integrated approach by combining information entropy theory and the rough number method to calculate the relative weights of decision criteria. Then a rough number based composite performance value is calculated to rank the candidate design concepts. By jointly applying the rough entropy weight method and rough composite performance values, both criteria weighing and alternative ranking can be manipulated without any auxiliary information. Thus, the proposed rough entropy based method can effectively reflect the decision makers' true perception and enhance the objectivity of design concept evaluation. The framework of the proposed method is depicted in Fig. 1.

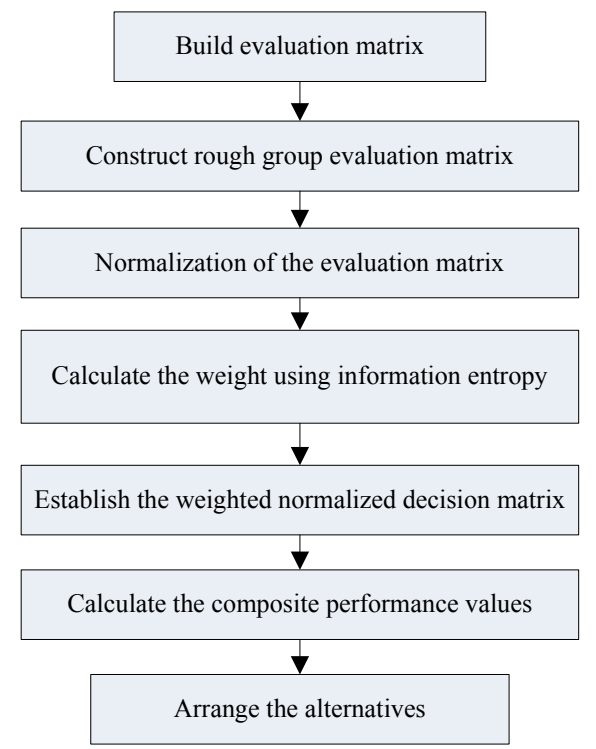

Figure 1. Framework of the proposed evaluation method

\section{B. Rough number based entropy weight method}

As a famous objective criteria weighting method, information entropy weight is widely used in various decision-making problems, especially in the situation where the weighting information is completely unknown. To deal with the vagueness and subjectivity in design concept evaluation, this paper introduces rough number in combination with the entropy weight method to aggregate individual judgments and conduct criteria weighing. The procedure of applying the rough entropy method is described as follows.

Step 1: Collect individual evaluation values and construct a group of decision matrices. The decision matrix of the $k$ th expert is described as:

$$
D_{k}=\left[\begin{array}{cccc}
x_{11}^{k} & x_{12}^{k} & \cdots & x_{1 n}^{k} \\
x_{21}^{k} & x_{22}^{k} & \cdots & x_{2 n}^{k} \\
\vdots & \vdots & \ddots & \vdots \\
x_{m 1}^{k} & x_{m 2}^{k} & \cdots & x_{m n}^{k}
\end{array}\right]
$$

where $x_{i j}^{k}(1 \leq i \leq m, 1 \leq j \leq n, 1 \leq k \leq s)$ is the evaluation value of criterion $j$ for alternative $i$ given by expert $k, m$ is the number of alternatives, $n$ is the number of criteria, and $s$ is the number of experts involved.

Then the integrated decision matrix $D$ is constructed as:

$$
\boldsymbol{D}=\left[\begin{array}{llll}
\tilde{x}_{11} & \tilde{x}_{12} & \cdots & \tilde{x}_{1 n} \\
\tilde{x}_{21} & \tilde{x}_{22} & \cdots & \tilde{x}_{2 n} \\
\vdots & \vdots & \ddots & \vdots \\
\tilde{x}_{m 1} & \tilde{x}_{m 2} & \cdots & \tilde{x}_{m n}
\end{array}\right]
$$

where $\tilde{x}_{i j}=\left\{x_{i j}^{1}, x_{i j}^{2}, \cdots, x_{i j}^{s}\right\}$, is the sequence of evaluation values of criterion $j$ on alternative $i$.

Step 2: Construct a rough decision matrix.

Translate the element $x_{i j}^{k}$ in $\tilde{x}_{i j}$ into rough number $R N\left(x_{i j}^{k}\right)$ using (1)-(6):

$$
R N\left(x_{i j}^{k}\right)=\left\lceil x_{i j}^{k L}, x_{i j}^{k U}\right\rfloor
$$

where $x_{i j}^{k L}$ is the lower limit of $R N\left(x_{i j}^{k}\right)$ while $x_{i j}^{k U}$ is the upper limit.

The rough sequence $R N\left(\tilde{x}_{i j}\right)$ is then represented as:

$$
R N\left(\tilde{x}_{i j}\right)=\left\{\left\lceil x_{i j}^{1 L}, x_{i j}^{1 U}\right\rfloor,\left\lceil x_{i j}^{2 L}, x_{i j}^{2 U}\right\rfloor, \cdots,\left\lceil x_{i j}^{s L}, x_{i j}^{s U}\right\rfloor\right\}
$$

It is further translated into an average rough number $R N\left(x_{i j}\right)$ by using rough arithmetic (8)-(10):

$$
\begin{aligned}
& R N\left(x_{i j}\right)=\left\lceil x_{i j}^{L}, x_{i j}^{U}\right\rfloor \\
& x_{i j}^{L}=\frac{x_{i j}^{1 L}+x_{i j}^{2 L}+\cdots+x_{i j}^{s L}}{s}
\end{aligned}
$$




$$
x_{i j}^{U}=\frac{x_{i j}^{1 U}+x_{i j}^{2 U}+\cdots+x_{i j}^{s U}}{s}
$$

where $x_{i j}^{L}$ is the lower limit of $R N\left(x_{i j}\right)$ and $x_{i j}^{U}$ is the upper limit.

Consequently, the rough decision matrix $D$ is formed as:

$$
D=\left[\begin{array}{cccc}
\left\lceil x_{11}^{L}, x_{11}^{U}\right\rfloor & \left\lceil x_{12}^{L}, x_{12}^{U}\right\rfloor & \ldots & \left\lceil x_{1 n}^{L}, x_{1 n}^{U}\right\rfloor \\
\left\lceil x_{21}^{L}, x_{21}^{U}\right\rfloor & \left\lceil x_{22}^{L}, x_{22}^{U}\right\rfloor & \ldots & \left\lceil x_{1 n}^{L}, x_{1 n}^{U}\right\rfloor \\
\vdots & \vdots & \ddots & \vdots \\
\left\lceil x_{m 1}^{L}, x_{m 1}^{U}\right\rfloor & \left\lceil x_{m 2}^{L}, x_{m 2}^{U}\right\rfloor & \ldots & \left\lceil x_{m n}^{L}, x_{m n}^{U}\right\rfloor
\end{array}\right]
$$

Step 3: Normalization

For making comparisons between different values, the elements of the decision matrix should be normalized first. For the benefit criterion:

$$
\begin{aligned}
& r_{i j}^{L}=\left[x_{i j}^{L}-\min _{i}\left(x_{i j}^{L}\right)\right] /\left[\max _{i}\left(x_{i j}^{U}\right)-\min _{i}\left(x_{i j}^{L}\right)\right] \\
& r_{i j}^{U}=\left[x_{i j}^{U}-\min _{i}\left(x_{i j}^{L}\right)\right] /\left[\max _{i}\left(x_{i j}^{U}\right)-\min _{i}\left(x_{i j}^{L}\right)\right]
\end{aligned}
$$

For the cost criterion:

$$
\begin{aligned}
r_{i j}^{L} & =\left[\max _{i}\left(x_{i j}^{U}\right)-x_{i j}^{U}\right] /\left[\max _{i}\left(x_{i j}^{U}\right)-\min _{i}\left(x_{i j}^{L}\right)\right] \\
r_{i j}^{L} & =\left[\max _{i}\left(x_{i j}^{U}\right)-x_{i j}^{L}\right] /\left[\max _{i}\left(x_{i j}^{U}\right)-\min _{i}\left(x_{i j}^{L}\right)\right]
\end{aligned}
$$

After normalization, a normalized decision matrix $R=\left[r_{i j}\right]_{m \times n}$ is obtained.

Step 4: Calculate the entropy of $R$ :

$$
\begin{gathered}
E_{j}^{L}=-k \sum_{i=1}^{m} f_{i j}^{L} \ln \left(f_{i j}^{L}\right) \\
E_{j}^{U}=-k \sum_{i=1}^{m} f_{i j}^{U} \ln \left(f_{i j}^{U}\right)
\end{gathered}
$$

where $f_{i j}^{L}=r_{i j}^{L} / \sum_{i=1}^{m} r_{i j}^{U}, f_{i j}^{U}=r_{i j}^{U} / \sum_{i=1}^{m} r_{i j}^{U}, k=1 / \ln n$, supposing $f_{i j}=0, f_{i j} \ln f_{i j}=0$.

The weight for the $j$ th criterion is defined as:

$$
\begin{gathered}
w_{j}^{L}=\frac{1-E_{j}^{U}}{\sum_{j=1}^{n}\left(1-E_{j}^{L}\right)} \\
w_{j}^{U}=\frac{1-E_{j}^{L}}{\sum_{j=1}^{n}\left(1-E_{j}^{L}\right)}
\end{gathered}
$$

Step 5: Rank the candidates using composite performance values which are calculated by:

$$
\begin{gathered}
I_{i}^{L}=\sum_{j=1}^{n} w_{j}^{L} r_{i j}^{L} \\
I_{i}^{U}=\sum_{j=1}^{n} w_{j}^{U} r_{i j}^{U}
\end{gathered}
$$

where $w_{j}$ is the normalized form of $w_{j}^{\prime}$.

The alternatives are arranged in an ascending order according to the composite performance values obtained. The alternative with the biggest composite performance value is the best one. The ranking rule of the interval numbers is described as follows [22]:

1) If $\underline{\operatorname{Lim}}(a) \geq \underline{\operatorname{Lim}}(b)$ and $\overline{\operatorname{Lim}}(a)>\overline{\operatorname{Lim}}(b)$, or $\underline{\operatorname{Lim}}(a)>\underline{\operatorname{Lim}}(b)$ and $\overline{\operatorname{Lim}}(a) \geq \overline{\operatorname{Lim}}(b), R N(a)>R N(b)$;

2) If $\underline{\operatorname{Lim}}(a)=\underline{\operatorname{Lim}}(b)$ and $\overline{\operatorname{Lim}}(a)=\overline{\operatorname{Lim}}(b)$, $R N(a)=R N(b)$;

3) If $\underline{\operatorname{Lim}}(b)>\underline{\operatorname{Lim}}(a)$ and $\overline{\operatorname{Lim}}(b)<\overline{\operatorname{Lim}}(a)$, or if $\underline{\operatorname{Lim}}(a)>\underline{\operatorname{Lim}}(b)$ and $\overline{\operatorname{Lim}}(a)<\overline{\operatorname{Lim}}(b)$, suppose $M(a)$ and $M(b)$ are the middle values of $R N(a)$ and $R N(b)$ :

a) If $M(a) \leq M(b)$, then $R N(a)<R N(b)$;

b) If $M(a)>M(b)$, then $R N(a)>R N(b)$;

By integrating information entropy theory and rough number, the concern on the subjectivity in the evaluation process can be effectively addressed.

\section{CASE STUDY}

\section{A. Design concept evaluation of an industrial robot}

In this section, the proposed rough entropy based method is used in the concept evaluation of an industrial robot to validate the effectiveness of its functions. Robots are widely used in the industrial environment to improve productivity, enhance product quality and execute special tasks. In industrial robot manufacturers, seven design concepts have been adopted in the conceptual design, namely, $\mathrm{A}_{1}, \mathrm{~A}_{2}, \mathrm{~A}_{3}$, $\mathrm{A}_{4}, \mathrm{~A}_{5}, \mathrm{~A}_{6}$ and $\mathrm{A}_{7}$.

The objective of the concept evaluation is to select the best alternative from the seven design concepts. Typically, decision makers are mainly concerned with the criteria such as: utility $\left(\mathrm{C}_{1}\right)$, manufacturability $\left(\mathrm{C}_{2}\right)$, novelty $\left(\mathrm{C}_{3}\right)$, power consumption $\left(\mathrm{C}_{4}\right)$, reliability $\left(\mathrm{C}_{5}\right)$, operability $\left(\mathrm{C}_{6}\right)$, structural complexity $\left(\mathrm{C}_{7}\right)$, and flexibility $\left(\mathrm{C}_{8}\right)$. Among them, $\mathrm{C}_{1}, \mathrm{C}_{2}$, $\mathrm{C}_{3}, \mathrm{C}_{5}, \mathrm{C}_{6}$, and $\mathrm{C}_{8}$ are the benefit criteria while $\mathrm{C}_{4}$ and $\mathrm{C}_{7}$ are the cost criteria. Three decision makers are asked to provide their opinions independently in the evaluation process. Fig. 2 illustrates the hierarchical structure of the design concept evaluation of the industrial robot.

In this evaluation, the rough entropy method is used to aggregate the individual judgments and calculate the corresponding criteria weights in the following steps.

Step 1: Collect individual judgments and construct a group of individual decision matrices, as follows: 


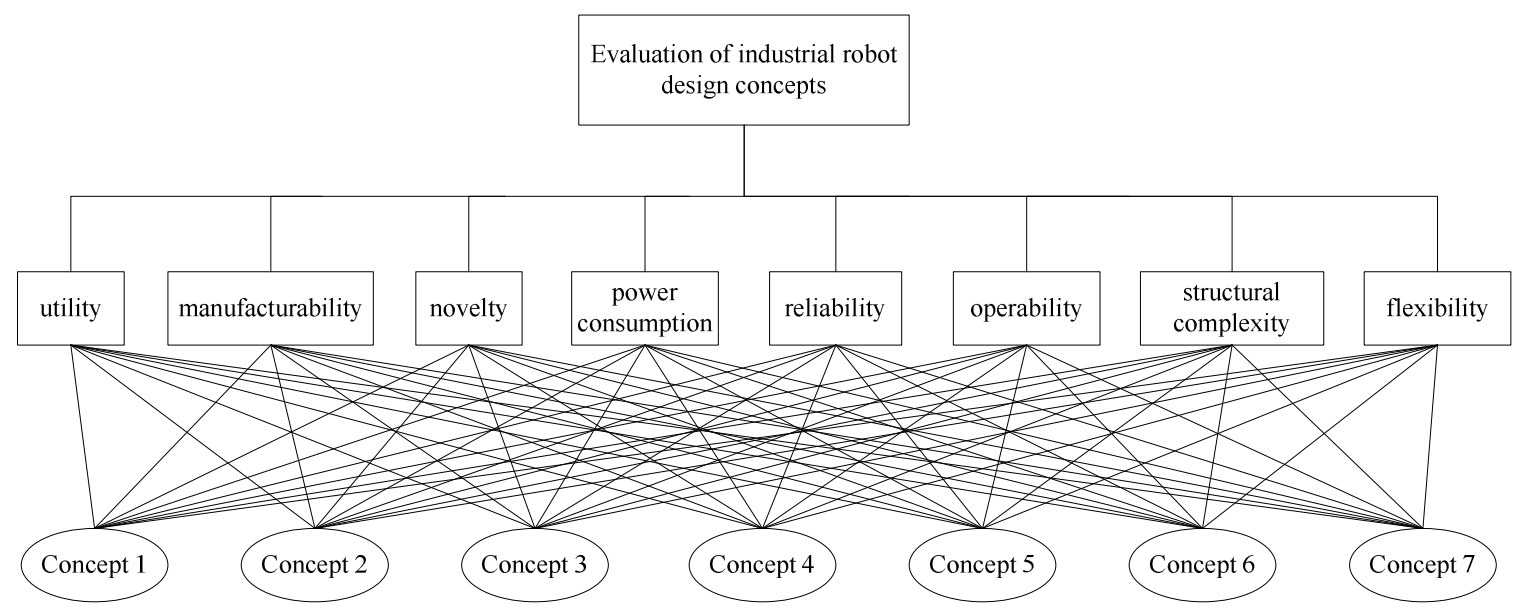

Figure 2. The hierarchical structure of the industrial robot design concept evaluation.

$$
D_{1}=\left[\begin{array}{llllllll}
3 & 5 & 7 & 7 & 5 & 5 & 3 & 1 \\
5 & 5 & 3 & 7 & 3 & 3 & 5 & 3 \\
7 & 9 & 9 & 5 & 7 & 7 & 5 & 7 \\
7 & 7 & 5 & 3 & 5 & 5 & 7 & 5 \\
9 & 5 & 5 & 7 & 5 & 3 & 9 & 1 \\
5 & 7 & 7 & 5 & 5 & 7 & 7 & 3 \\
3 & 5 & 5 & 7 & 3 & 3 & 7 & 5
\end{array}\right]
$$$$
D_{2}=\left[\begin{array}{llllllll}
5 & 5 & 9 & 7 & 3 & 5 & 5 & 3 \\
7 & 5 & 5 & 7 & 3 & 5 & 7 & 1 \\
9 & 7 & 9 & 3 & 7 & 7 & 7 & 9 \\
7 & 5 & 7 & 5 & 7 & 5 & 7 & 5 \\
7 & 7 & 7 & 9 & 5 & 5 & 9 & 3 \\
5 & 7 & 5 & 7 & 7 & 5 & 5 & 5 \\
3 & 3 & 5 & 5 & 3 & 5 & 5 & 5
\end{array}\right]
$$$$
D_{3}=\left[\begin{array}{llllllll}
5 & 7 & 7 & 9 & 5 & 3 & 3 & 3 \\
5 & 7 & 5 & 9 & 3 & 5 & 7 & 3 \\
7 & 9 & 9 & 3 & 7 & 9 & 5 & 9 \\
5 & 7 & 7 & 5 & 5 & 7 & 5 & 5 \\
7 & 9 & 5 & 9 & 3 & 5 & 9 & 3 \\
7 & 5 & 5 & 7 & 5 & 7 & 5 & 3 \\
5 & 3 & 3 & 5 & 5 & 3 & 7 & 5
\end{array}\right]
$$

Then an integrated decision matrix $D$ is generated by combining the individual decision matrices formed.

$$
D=\left[\begin{array}{ccccc}
3,5,5 & 5,5,7 & \ldots & 3,5,3 & 1,3,3 \\
5,7,5 & 5,5,7 & \ldots & 5,7,7 & 3,1,3 \\
7,9,7 & 9,7,9 & \ldots & 5,7,5 & 7,9,9 \\
7,7,5 & 7,5,7 & \ldots & 7,7,5 & 5,5,5 \\
9,7,7 & 5,7,9 & \ldots & 9,9,9 & 1,3,3 \\
5,5,7 & 7,7,5 & \ldots & 7,5,5 & 3,5,3 \\
3,3,5 & 5,3,3 & \ldots & 7,5,7 & 5,5,5
\end{array}\right]
$$

Step 2: Translate the elements in $D$ into rough numbers and then the original integrated decision matrix is converted into a rough decision matrix according to (15)-(17).

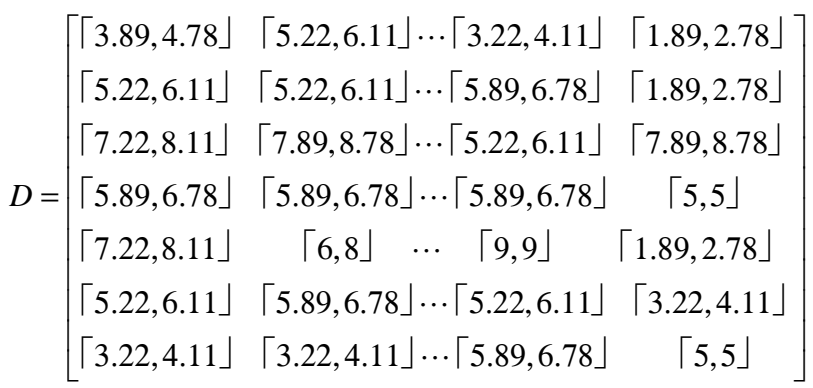

Step 3: Conduct the normalization on the rough decision matrix is conducted according to (19)-(22) and the normalized decision matrix is obtained as:

$$
R=\left[\begin{array}{cccc}
\lceil 0.14,0.32\rfloor & \lceil 0.36,0.52\rfloor \ldots\lceil 0.85,1\rfloor & \lceil 0,0.13\rfloor \\
\lceil 0.41,0.59\rfloor & \lceil 0.36,0.52\rfloor \ldots\lceil 0.38,0.54\rfloor & \lceil 0,0.13\rfloor \\
\lceil 0.82,1\rfloor & \lceil 0.84,1\rfloor \ldots\lceil 0.5,0.65\rfloor & \lceil 0.87,1\rfloor \\
\lceil 0.55,0.73\rfloor & \lceil 0.48,0.64\rfloor \ldots\lceil 0.38,0.54\rfloor & \lceil 0.45,0.45\rfloor \\
\lceil 0.82,1\rfloor & \lceil 0.5,0.86\rfloor \ldots & \lceil 0,0\rfloor & \lceil 0,0.13\rfloor \\
\lceil 0.41,0.59\rfloor & \lceil 0.48,0.64\rfloor \ldots\lceil 0.5,0.65\rfloor & \lceil 0.19,0.32\rfloor \\
\lceil 0,0.18\rfloor & \lceil 0,0.16\rfloor \ldots & \lceil 0.38,0.54\rfloor & \lceil 0.45,0.45\rfloor
\end{array}\right]
$$


Step 4: Calculate rough entropy weights used as the evaluation criteria and the entropy of the evaluation criteria is obtained using (23)-(24), as illustrated in Table I.

TABLE I. ENTROPY VALUES $\left\lceil E_{i}^{L}, E_{i}^{U}\right\rfloor$

\begin{tabular}{cccc}
\hline & $E_{i}^{L}$ & $E_{i}^{U}$ & $E_{i}$ \\
\hline $\mathrm{C}_{1}$ & 0.691 & 0.880 & $\lceil 0.691,0.880\rfloor$ \\
$\mathrm{C}_{2}$ & 0.705 & 0.891 & $\lceil 0.705,0.891\rfloor$ \\
$\mathrm{C}_{3}$ & 0.618 & 0.857 & $\lceil 0.618,0.857\rfloor$ \\
$\mathrm{C}_{4}$ & 0.653 & 0.860 & $\lceil 0.653,0.860\rfloor$ \\
$\mathrm{C}_{5}$ & 0.635 & 0.822 & $\lceil 0.635,0.822\rfloor$ \\
$\mathrm{C}_{6}$ & 0.613 & 0.867 & $\lceil 0.613,0.867\rfloor$ \\
$\mathrm{C}_{7}$ & 0.738 & 0.848 & $\lceil 0.738,0.848\rfloor$ \\
$\mathrm{C}_{8}$ & 0.559 & 0.808 & $\lceil 0.559,0.808\rfloor$ \\
\hline
\end{tabular}

The rough entropy weights are obtained using (25)-(26):

$$
\begin{aligned}
w^{\prime}= & \left\{w_{1}^{\prime}, w_{2}^{\prime}, w_{3}^{\prime}, w_{4}^{\prime}, w_{5}^{\prime}, w_{6}^{\prime}, w_{7}^{\prime}, w_{8}^{\prime}\right\} \\
= & \{\lceil 0.043,0.111\rfloor,\lceil 0.039,0.106\rfloor,\lceil 0.051,0.137\rfloor, \\
& \lceil 0.050,0.125\rfloor,\lceil 0.064,0.131\rfloor,\lceil 0.048,0.139\rfloor, \\
& \lceil 0.055,0.094\rfloor,\lceil 0.069,0.158\rfloor\}
\end{aligned}
$$

The normalized format of $w$ is then obtained, as listed in Table II.

TABLE II. ENTROPy WeIGHT VALUES $\left\lceil w_{i}^{L}, w_{i}^{U}\right\rfloor$

\begin{tabular}{cccc}
\hline & $w_{i}^{L}$ & $w_{i}^{U}$ & $w_{i}$ \\
\hline $\mathrm{C}_{1}$ & 0.272 & 0.703 & $\lceil 0.272,0.703\rfloor$ \\
$\mathrm{C}_{2}$ & 0.247 & 0.671 & $\lceil 0.247,0.671\rfloor$ \\
$\mathrm{C}_{3}$ & 0.323 & 0.867 & $\lceil 0.323,0.867\rfloor$ \\
$\mathrm{C}_{4}$ & 0.317 & 0.791 & $\lceil 0.317,0.791\rfloor$ \\
$\mathrm{C}_{5}$ & 0.405 & 0.829 & $\lceil 0.405,0.829\rfloor$ \\
$\mathrm{C}_{6}$ & 0.304 & 0.880 & $\lceil 0.304,0.880\rfloor$ \\
$\mathrm{C}_{7}$ & 0.348 & 0.595 & $\lceil 0.348,0.595\rfloor$ \\
$\mathrm{C}_{8}$ & 0.437 & 1 & $\lceil 0.437,1\rfloor$ \\
\hline
\end{tabular}

Step 5: Rank the alternatives using composite performance values according to (27)-(28), as shown in Table III.

TABLE III. Composite Performance Values $\left\lceil I_{i}^{L}, I_{i}^{U}\right\rfloor$

\begin{tabular}{ccccc}
\hline & $I_{i}^{L}$ & $I_{i}^{U}$ & $I_{i}$ & Rank \\
\hline $\mathrm{A}_{1}$ & 0.803 & 2.895 & $\lceil 0.803,2.895\rfloor$ & 4 \\
$\mathrm{~A}_{2}$ & 0.413 & 1.866 & $\lceil 0.413,1.866\rfloor$ & 7 \\
$\mathrm{~A}_{3}$ & 2.228 & 6.128 & $\lceil 2.228,6.128\rfloor$ & 1 \\
$\mathrm{~A}_{4}$ & 1.303 & 4.070 & $\lceil 1.303,4.070\rfloor$ & 2 \\
$\mathrm{~A}_{5}$ & 0.562 & 2.564 & $\lceil 0.562,2.564\rfloor$ & 5 \\
$\mathrm{~A}_{6}$ & 1.079 & 3.624 & $\lceil 1.079,3.624\rfloor$ & 3 \\
$\mathrm{~A}_{7}$ & 0.505 & 2.049 & $\lceil 0.505,2.049\rfloor$ & 6 \\
\hline
\end{tabular}

According to the ranking rules stated in Section III, the final ranking of the candidate alternatives is obtained as follows: $A_{2}<A_{7}<A_{5}<A_{1}<A_{6}<A_{4}<A_{3}$. Obviously, alternative $\mathrm{A}_{3}$ is the best design concept in this case.

\section{B. Comparison and discussion}

To validate the performance of the proposed rough entropy based (rough-entropy) method, traditional crisp entropy weight (crisp-entropy) method is performed on the same data stated above. Fig. 3 shows the comparison of alternative ranking calculated by the crisp-entropy method and the proposed rough-entropy method.

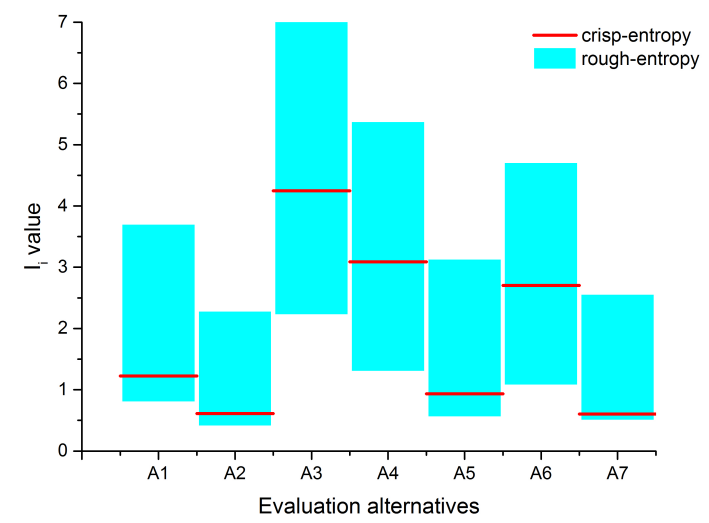

Figure 3. Comparison of the alternative ranking

From Fig. 3, we can see that the final ranking calculated by the crisp entropy weight method is $\mathrm{A}_{7}<\mathrm{A}_{2}<\mathrm{A}_{5}<$ $\mathrm{A}_{1}<\mathrm{A}_{6}<\mathrm{A}_{4}<\mathrm{A}_{3}$ while $\mathrm{A}_{2}<\mathrm{A}_{7}<\mathrm{A}_{5}<\mathrm{A}_{1}<\mathrm{A}_{6}<\mathrm{A}_{4}<\mathrm{A}_{3}$ is the result of the rough entropy method. Actually, the differences between $A_{2}$ and $A_{7}$ are subtle. For both methods, alternative $\mathrm{A}_{3}$ is the best design concept. However, the integrated rough entropy based method uses the original decision data only to carry out concept evaluation, without any auxiliary information. The rough number can naturally aggregate and translate the crisp evaluation numbers into the interval numbers without needing any additional tools. It adopts a flexible interval boundary instead of a fixed predefined one, which denotes the vagueness of the decision results. Therefore, the proposed rough entropy based method can effectively reflect the decision makers' true perception and enhance the objectivity of decision making for design concept evaluation.

\section{CONCLUSION}

To manipulate the subjectivity and vagueness in design concept evaluation, this paper proposes an integrated rough entropy based method to strengthen the objectivity and credibility of the evaluation process. Rough number is adopted here to aggregate individual judgments and preferences. First of all, it is applied to combine with the entropy weight method to compute criteria weights. Rough number based composite performance values are then calculated to arrange the candidate alternatives. By integrating rough number and an entropy weight method, the problems regarding vagueness and subjectivity in design concept evaluation can be properly addressed. The relative importance of the evaluation criterion is calculated using the 
entropy weight method which is an objective method to eliminate the bias of the decision maker. This approach is especially useful in the situation where the information about the relative weight is completely unknown. Finally, the proposed rough entropy based method is applied in a design concept evaluation of an industrial robot. The case study results have confirmed that the rough entropy based method can effectively reflect the true perception of the decision maker. It is also shown that the vagueness of the original information can be properly manipulated and the results obtained are more objectively represented.

The proposed rough number based approach is also applicable to many other group decision-making areas, which will be pursued in our future work.

\section{ACKNOWLEDGMENT}

This research is supported by the National Natural Science Foundation of China (No. 51475288, 51275293, 51305260), National Key Scientific Instruments and Equipment Development Program of China (No. 2011YQ030114, 2013YQ03065105), "Shu Guang" project supported by Shanghai Municipal Education Commission and Shanghai Education Development Foundation (12SG14), Shanghai Committee of Science and Technology (No. 13111102800), Natural Science Foundation of Shanghai (No. 13ZR1421400).

\section{REFERENCES}

[1] R.V. Rao, Decision Making in Manufacturing Environment Using Graph Theory and Fuzzy Multiple Attribute Decision Making Methods, vol. 2, Berlin: Springer, 2012.

[2] J. Yang and W. Qiu, A measure of risk and a decision-making model based on expected utility and entropy, European Journal of Operational Research, 164(3), 2005, pp. 792-799.

[3] X. Li, K. Wang, L. Liu, J. Xin, H. Yang, and C. Gao, Application of the entropy weight and TOPSIS method in safety evaluation of coal mines, Procedia Engineering, 26, 2011, pp. 2085-2091.

[4] M. F. El-Santawy, A VIKOR Method for Solving Personnel Training Selection Problem, International Journal of Computing Science, 1(2), 2012, pp. 9-12.

[5] H. Safari, M. S. Fagheyi, S. S. Ahangari, and M. R. Fathi, Applying PROMETHEE method based on entropy weight for supplier selection, Business management and strategy, 3(1), 2012, pp. 97-106.

[6] P. Liu and X. Zhang, Research on the supplier selection of a supply chain based on entropy weight and improved ELECTRE-III method, International Journal of Production Research, 49(3), 2011, pp. 637 646.

[7] P. Li, J. Wu, and H. Qian, Groundwater quality assessment based on entropy weighted osculating value method, International Journal of Environmental Sciences, 1(4), 2010, pp. 621-630.
[8] O. Kulak, A decision support system for fuzzy multi-attribute selection of material handling equipments, Expert systems with applications, 29(2), 2005, pp. 310-319.

[9] D. Akay and O. Kulak, Evaluation of product design concepts using grey-fuzzy information axiom, Journal of Grey Systems, 19(3), 2007, pp. 221-234.

[10] G. Büyüközkan, An integrated fuzzy multi-criteria group decisionmaking approach for green supplier evaluation, International Journal of Production Research, 50(11), 2012, pp. 2892-2909.

[11] D. Akay, O. Kulak, and B. Henson, Conceptual design evaluation using interval type-2 fuzzy information axiom, Computers in Industry, 62(2), 2011, pp. 138-146.

[12] F. T. Weng, S. M. Jenq, Application integrating axiomatic design and agile manufacturing unit in product evaluation, The International Journal of Advanced Manufacturing Technology, 63(1-4), 2012, pp. 181-189.

[13] S. Cebi and C. Kahraman, Developing a group decision support system based on fuzzy information axiom, Knowledge-Based Systems, 23(1), 2010, pp. 3-16.

[14] O. Kulak and C. Kahraman, Fuzzy multi-attribute selection among transportation companies using axiomatic design and analytic hierarchy process, Information Sciences, 170(2), 2005, pp. 191-210.

[15] Y. Ji, G. H. Huang, and W. Sun, Risk assessment of hydropower stations through an integrated fuzzy entropy-weight multiple criteria decision making method: A case study of the Xiangxi River, Expert Systems with Applications (2015), http://dx.doi.org/10.1016/ j.eswa. 2014.12.026.

[16] L. Liu, J. Zhou, X. An, Y. Zhang, and L. Yang, Using fuzzy theory and information entropy for water quality assessment in Three Gorges region, China, Expert Systems with Applications, 37(3), 2010, pp. 2517-2521.

[17] T. Y. Chen and C. H. Li, Determining objective weights with intuitionistic fuzzy entropy measures: a comparative analysis, Information Sciences, 180(21), 2010, pp. 4207-4222.

[18] Z. Zhao, M. Qi, Y. Zhang, and X. Xiao, Reliability Evaluation of Power Communication Network Based on FAHP and Entropy Method, Intelligent Systems and Applications (ISA), 2010 2nd International Workshop on. IEEE, 2010, pp. 1-4.

[19] J. Ye, Multicriteria fuzzy decision-making method using entropy weights-based correlation coefficients of interval-valued intuitionistic fuzzy sets, Applied Mathematical Modelling, 34(12), 2010, pp. 38643870 .

[20] J Ye, Fuzzy decision-making method based on the weighted correlation coefficient under intuitionistic fuzzy environment, European Journal of Operational Research, 205(1), 2010, pp. 202204.

[21] L.-Y. Zhai, L.-P. Khoo, and Z.-W. Zhong, Design concept evaluation in product development using rough sets and grey relation analysis, Expert Systems with Applications, 36(3), 2009, pp. 7072-7079.

[22] L.-Y. Zhai, L.-P. Khoo, and Z.-W. Zhong, A rough set enhanced fuzzy approach to quality function deployment, The International Journal of Advanced Manufacturing Technology, 37(5-6), 2008, pp. 613-624.

[23] L.-Y. Zhai, L.-P. Khoo, and Z.-W. Zhong, A rough set based QFD approach to the management of imprecise design information in product development, Advanced Engineering Informatics, 23(2), 2009, pp. 222-228. 